\title{
铁催化二级氯代烷烃与炔基格氏试剂的交叉偶联反应
}

\author{
贾 婉 ${ }^{a}$ 赵立志 ${ }^{b}$ 魏恒旭 ${ }^{b}$ 朱林东 ${ }^{b}$ 傅 䂞 ${ }^{a}$ 陈蔚春 $*, b$ \\ $\left({ }^{a}\right.$ 上海交通大学药学院 上海 200240) \\ ( ${ }^{b}$ 葛兰素史克(上海)医药研发有限公司 上海 201203)
}

\begin{abstract}
摘要 一个简单高效地制备烷基炔的方法被开发. 它以廉价绿色的三氯化铁为催化剂, 使低活性的二级氯代烷烃与炔 基格氏试剂顺利发生交叉偶联反应. 该方法收率高、适用范围广、反应迅速、操作简便、不需要有机碱辅助，可替代 钯或镍的催化作用, 为合成烷基炔化合物提供了一条绿色途径, 具备良好的工业化发展前景.

关键词 铁催化; 二级氯代烷烃; 炔基格氏试剂; 交叉偶联反应; 绿色化学
\end{abstract}

\section{Iron-Catalyzed Cross-Coupling of Secondary Alkyl Chloride and the Alkynes Grignard Reagent}

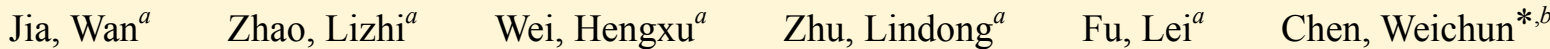 \\ ( School of Pharmacy, Shanghai Jiao Tong University, Shanghai 200240) \\ ( ${ }^{b}$ Research and Developemnt Center in Shanghai of GlaxoSmithKline, Shanghai 201203)
}

\begin{abstract}
A green, practical and efficient iron-catalyzed, base free cross-coupling reaction of non-activated secondary alkyl halides with the alkynes Grignard reagent has been developed to afford a variety of alkyl alkynes in high yields, using iron(III) chlorides as catalyst. This approach is quick and easy to operate. It exhibits promising in industry since utilizing the low-cost iron as a catalyst instead of expensive palladium or nickel.

Keywords iron-catalyzed; secondary alkyl choloride; alkynes Grignard reagents; cross-coupling reactions; green chemistry
\end{abstract}

碳碳键的构建是有机合成中非常有用的反应类型, 最经典的方法是过渡金属催化的交叉偶联反应. 比如 Negishi 反应 ${ }^{[1]}$ S Suzuki 反应 ${ }^{[2]}$ 、Sonogashira 反应 ${ }^{[3]}$ 以及 Heck 反应 ${ }^{[4]}$ 等. 这些反应使用钯、钴、镍、铜等过渡金 属作为催化剂, 具有高收率、强底物适应性和官能团容 忍性等特点. 但是, 昂贵的金属催化剂及其不可忽视的 人体毒理性以及繁复的重金属后处理过程都严重地阻 碍了这些方法在工业化生产中的应用 ${ }^{[5]}$. 为克服偶联反 应在工业生产中应用的重重困难, 许多科学家尝试将铁 与铜结合应用到碳碳键的构建中 ${ }^{[6]}$. 虽然在一定程度上 缓解了以往金属催化剂催化偶联反应时所存在的种种 弊端, 但是反应时间长、反应温度高等缺点使偶联反应 的催化体系仍存在着极大的改进空间 ${ }^{[7]}$.

自 19 世纪 80 年代起, 铁开始被越来越多的化学家 用来催化偶联反应 ${ }^{[8,9]}$. 但在接下来的 20 年内, 科学家
们并没有在铁对偶联反应的催化作用上给予许多关注. 近来, 绿色化学的兴起使廉价低毒的铁成为研究热点. 铁可以催化多种类型的偶联反应，如： $\mathrm{sp}^{2}-\mathrm{sp}^{3}{ }^{[10]} 、 \mathrm{sp}^{2}-$ $\mathrm{sp}^{2[1]]} \mathrm{sp}-\mathrm{sp}^{3[12 \sim 14]}$ 以及 $\mathrm{sp}^{3}-\mathrm{sp}^{3[15]}$ 等. 其中日本的 Nakamura 实验室的研究成果尤为显著 ${ }^{[16]}$. Nakamura 等 ${ }^{[17]}$ 使用三氯化铁催化缩醛与格氏试剂的不对称偶联, 产物 的对映体过量值可达 $95 \%$ 以上. Lautens 等 ${ }^{[18]}$ 在研究三 氯化铁的催化性能的过程中发现，在四甲基乙二胺 (TMEDA)作碱的条件下三氯化铁可催化氧杂双环烯烃 的开环反应, 生成对应的烯烃. Nakamura 实验室的研究 阐明了铁化合物的催化性能, 还预示着有机合成的新方 向.

尽管在科学家们的不解努力下, 人类在铁催化方面 的研究越来越全面, 但是对于碳碳键的构建还主要停留 在烷基溴/碘代物，对于惰性的氯代物没有很多深入的

\footnotetext{
* E-mail: weichun.w.chen@gsk.com

Received October 26, 2015; revised December 16, 2015; published online January 4, 2016.

Project supported by the Union-Education Program of Shanghai Jiao Tong University and the Research and Development Center of GlaxoSmithKline. 葛兰素史克(上海)医药研发有限公司与上海交通大学药学院联合培养项目.
} 
研究. 2011 年, Nakamura 实验室用自行合成的二价铁膦 配体化合物对非活化的卤代物与 4-溴-1-环己烯基-三氟 甲磺酸盐的偶联反应进行了催化 ${ }^{[11]}$, 以此来证明使用双 膦配体能够增大催化剂的立体选择性. 在实验过程中, 他们用二价铁双膦配合物催化了氯代环庚烷与三异丙 基硅基乙炔基格氏试剂的偶联，反应收率仅为 69\%. 然 而，这种方法没有被应用到更多种类的二级氯代烷烃与 炔的偶联中. 2014 年, Cheung 等 ${ }^{[13]}$ 用 $\mathrm{FeBr}_{2}$ 在没有配体 和室温的条件下成功地催化了溴代与碘代二级烷烃与 炔基格氏试剂的偶联. 该方法与 Nakamura 实验室的方 法相比有改进的地方, 也尝试了多种类型的二级溴代或 碘代烷烃, 收率可达 95\%. 但是, 该方法并没有涉及广 泛存在但活性低的二级氯代物. 可见, 开发出一种以二 级氯代烷烃为底物的 Kumada 偶联反应合成烷基炔是必 要的.

本文报道了一种铁催化的二级氯代烷烃与炔基格 氏试剂偶联合成烷基炔的新方法, 去掉了以往偶联反应 过程中常用到的碱. 该方法反应迅速、收率高、底物范 围广、操作简便、不需要有机碱辅助, 为合成烷基炔化 合物提供了一条绿色高效的途径.

\section{1 结果与讨论}

\section{1 反应条件的优化}

首先, 我们以氯代环庚烷(1a)与三异丙基硅基乙炔 基溴化镁(1b)的偶联反应为模板. 以 $\mathrm{FeCl}_{3}$ 为催化剂、

TMEDA 为碱、Xantphos 为配体、甲苯为溶剂, 将上述 反应组分一次性加入到可封闭的耐压试管中，在 $100{ }^{\circ} \mathrm{C}$ 的温度下反应 $3 \mathrm{~h}$ 即可获得理想产物 1c. 然而, 所得收率仅为 $43 \%$, 转化率为 $74 \%$ (Eq. 1). 我们尝试改 变加样顺序, 先将 $\mathrm{FeCl}_{3}$ 和 Xantphos 一起加入到甲苯中, 搅拌一段时间后再加入碱和原料, 反应收率可提升到 $62 \%$, 并且能够完全转化. 在这个过程中, $\mathrm{FeCl}_{3}$ 可能与 Xantphos 结合形成了 II 价的配合物, 从而提高了催化性 能. 这与 Nakamura 实验室的研究思路是一致的 ${ }^{[12]}$.

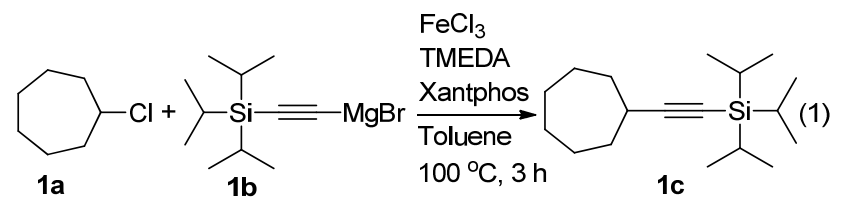

基于上述实验结果, 我们对反应条件展开了优化, 考察了溶剂、温度、碱以及配体等对反应的影响(表 1). 首先, 反应体系中的溶剂被篎选, 如甲苯(Toluene)、1,4二氧六环(1,4-Dioxane)、甲基四氢呋喃(Me-THF)、 $N$-甲 基吡咯烷酮(NMP)、丙酮(ACE)和乙腈(ACN). 其中, 以 甲基四氢呋喃为溶剂时, 收率可提高至 $63 \%$ (如表 1 ,
表 1 反应条件的优化 ${ }^{a}$

Table 1 The optimization of the reaction conditions

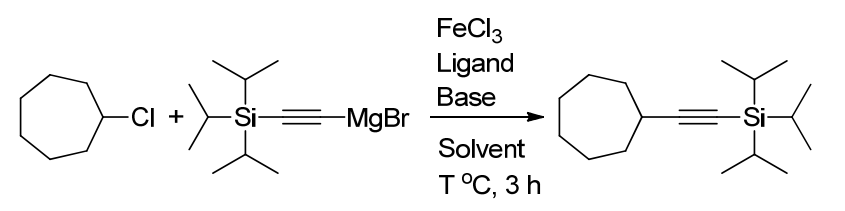

\begin{tabular}{clllcc}
\hline Entry $^{a}$ & \multicolumn{1}{c}{ Base } & \multicolumn{1}{c}{ Solvent } & Ligand & $T /^{\circ} \mathrm{C}$ & Yield $^{b} \%$ \\
\hline 1 & TMEDA & Toluene & Xantphos & 100 & 62 \\
2 & TMEDA & 1,4-Dioxane & Xantphos & 100 & 50 \\
3 & TMEDA & Me-THF & Xantphos & 100 & 63 \\
4 & TMEDA & NMP & Xantphos & 100 & 12 \\
5 & TMEDA & ACE & Xantphos & 100 & 0 \\
6 & TMEDA & ACN & Xantphos & 100 & 3 \\
7 & None & Me-THF & Xantphos & 100 & 73 \\
8 & DABCO & Me-THF & Xantphos & 100 & 8 \\
& N-Methyl & & & & \\
9 & Me-THF & Xantphos & 100 & 64 \\
10 & Quinoline & Me-THF & Xantphos & 100 & 59 \\
11 & DBU & Me-THF & Xantphos & 100 & 53 \\
12 & Cs ${ }_{2}$ CO ${ }_{3}$ & Me-THF & Xantphos & 100 & 6 \\
13 & None & THF & Xantphos & 100 & 74 \\
14 & None & TBME & Xantphos & 100 & 69 \\
15 & None & THF & None & 100 & 58 \\
16 & None & THF & Dppe & 100 & 14 \\
17 & None & THF & Dppf & 100 & 49 \\
18 & None & THF & BINAP & 100 & 35 \\
19 & None & THF & Dppp & 100 & 11 \\
20 & None & Me-THF & Xantphos & 80 & 71 \\
21 & None & Me-THF & Xantphos & 60 & 66 \\
\hline
\end{tabular}

${ }^{a}$ 反应条件: 1 equiv.氯代环庚烷、 2 equiv.炔基格式试剂、0.1 equiv. $\mathrm{FeCl}_{3}$ 、 0.2 equiv.配体、 2 equiv. $100 \mathrm{mg}$ 的原料, 溶剂 $1.0 \mathrm{~mL}$, 封管反应 $3 \mathrm{~h} ;{ }^{b}$ 以十 一烷作内标, 由 GCMS 定量检测所得.

Entries 1 6). 接着，在甲基四氢呋喃为溶剂的条件下， 一系列常用的有机碱和无机碱被考察，包括 1,4-二氮杂 二环 [2,2,2] 辛烷 (DABCO)、 $N$-甲基吗啡林 $(N$-methyl morpholine)、喹啉(Quinoline)、1,8-二氮杂二环十一碳-7烯(DBU)和碳酸铯 $\left(\mathrm{Cs}_{2} \mathrm{CO}_{3}\right)$, 并做空白对照. 结果显示, 当不添加碱时收率最高，可达 73\%(如表 1, Entries 7 12). 这一现象表明, 该交叉偶联反应无需碱的促进.

同时，我们推测含氧溶剂对反应体系有促进作用. 在去掉偶联反应体系中常用碱的情况下，四氢呋喃 (THF)和甲基叔丁基醚(TBME)等醚类溶剂也被尝试. 结 果显示用 THF 作溶剂时, 反应收率能够达到 74\%(表 1, Entries 13,14), 相比于 Me-THF 作溶剂时的收率稍有提 高. 紧接着, 在 THF 作溶剂的条件下, 我们对反应体系 中的配体进行进一步的篮选. 用 1,2 -双 (二苯膦) 乙烷 (dppe)、1, $1^{\prime}$-双 (二苯膦)二茂铁、联菜二苯磷(BINAP)以 及 1,3-双 (二苯基膦)丙烷(dppp)代替 Xantphos，并做空白 对照试验，反应收率均有不同程度的下降(如表 1, Entries 15 19). 基于绿色化学的理念, Me-THF 比 THF 更 
具有环境友好性, 于是, 我们仍选择 Me-THF 作为反应 体系的溶剂.

温度也是影响反应收率的一个重要影响因素. 在 Me-THF 作溶剂的条件下, 反应在 $100 、 80{ }^{\circ} \mathrm{C}$ 以及 $60{ }^{\circ} \mathrm{C}$ 下的收率分别为 $73 \%$ 、70\%以及 $66 \%$ (如表 1 , Entries 7 、 21、22), 呈现下降趋势. 考虑到 Me-THF 的回流温度只 有 $80{ }^{\circ} \mathrm{C}$, 去除封管, 我们对这个反应做了进一步验证. 结果显示, 在 Xantphos 作配体、Me-THF 作溶剂的条件 下, 反应在氮气流的保护下回流 $3 \mathrm{~h}\left(80{ }^{\circ} \mathrm{C}\right)$, 收率可进 一步提高到 $80 \%$ (表 2, Entry 1).

表 2 铁催化的二级氯代烷烃与炔基格氏试剂的偶联反应底 物拓展 ${ }^{a}$

Table 2 Iron-catalyzed the cross-coupling of secondly alkyl halides and the alkynes Grignard reagent: substrate scope

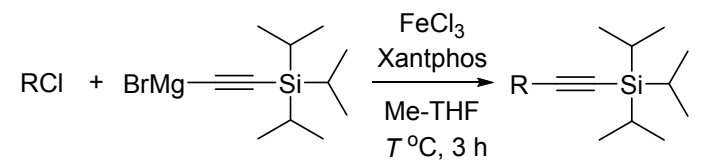

\begin{tabular}{|c|c|c|c|c|}
\hline Entry $^{a}$ & Substrate 1 & $T /{ }^{\circ} \mathrm{C}$ & Produ & eld $/ \%$ \\
\hline 1 & $1 a$ & $74^{b}$ & 1c & 80 \\
\hline 2 & $2 a$ & $73^{b}$ & $2 c$ & 79 \\
\hline 3 & $3 a$ & $71^{b}$ & $3 c$ & 77 \\
\hline 4 & $4 a$ & $70^{b}$ & $4 c$ & 61 \\
\hline 5 & $5 a$ & $71^{b}$ & $5 c$ & 78 \\
\hline $6^{d}$ & & 80 & $6 c$ & 85 \\
\hline $7^{d}$ & 7 & 80 & $7 c$ & 71 \\
\hline $8^{d}$ & & 80 & $8 c$ & 66 \\
\hline $9^{d}$ & & 80 & $9 c$ & 73 \\
\hline
\end{tabular}

厚应条件: 1 equiv.原料, 2 equiv.格氏试剂, 0.1 equiv. $\mathrm{FeCl}_{3}, 0.2$ equiv. Xantphos, Me-THF 为溶剂, 氮气流的保护下回流 $3 \mathrm{~h} ;{ }^{b}$ 内温; ${ }^{c}$ 分离产率, 基 于反应物的计算; ${ }^{d}$ 封管反应, 温度为外温.

最终我们确定最佳反应条件为: 1 equiv.原料, 0.1 equiv. $\mathrm{FeCl}_{3}$ 作催化剂, 0.2 equiv. Xantphos 作配体, $\mathrm{Me}-\mathrm{THF}$ 作溶剂以及 2 equiv.格氏试剂, 在 $80{ }^{\circ} \mathrm{C}$ 下回流 $3 \mathrm{~h}$.

\section{2 底物范围的扩展}

在上述优化的条件下，我们对铁催化的二级氯代烷 烃与炔基格氏试剂的偶联反应进行了底物扩展，如表 2 . 我们尝试了二级氯代环烷烃以及二级氯代氮杂/氧杂环 烷烃. 其中二级氯代环烷烃中的 $1 \mathrm{c} 、 2 \mathrm{c} 、 3 \mathrm{c}$ 的收率分别 能够达到 80\%、79\%、77\%(如表 2, Entries 1、2、3). 这 样的结果相对于 Nakamura 实验室的研究成果来说, 是 一个新的突破. 此外, 我们还尝试了简单易得的杂环化 合物 4a 和 5a, 并在此基础上合成具有不同官能团的杂 环化合物以扩大底物范围. 如 6a、7a、8a 和 9a(如表 2, Entries 4、5、6、7、8、9), 分别以 $61 \% 、 78 \% 、 85 \%$ 、 $71 \% 、 66 \%$ 和 $73 \%$ 的收率得到相应的偶联产物 $4 \mathbf{c} 、 5 \mathbf{c} 、$ 6c、7c、8c 和 $9 c$.

\section{2 结论}

我们发展了一种用 $\mathrm{FeCl}_{3}$ 和配体 Xantphos 结合来催 化二级氯代烷烃与炔基格式试剂进行反应以制得烷基 炔的方法. 该方法使烷基炔的制备不再依赖于烷基溴代 物和碘代物，而且反应收率高、操作简便、绿色环保，符 合绿色化学的要求，也符合可持续发展的理念. 关于该 方法在更多烷基炔合成中的应用，以及铁催化该类反应 的机制仍在探索中.

\section{3 实验部分}

\section{1 仪器与试剂}

实验所用溶剂使用前均按照处理溶剂的标准方法 进行, 所用铁催化剂从 Alfa Aesar 购买, 所用试剂从梯 希爱(上海)化成工业发展有限公司或百灵威科技有限公 司购买, 未经进一步纯化直接使用; 实验所用氮气无需 进一步干燥. 柱层析使用 Biotag 公司的不同质量规格的 正相色谱柱和 $\mathrm{C} 18$ 反相色谱柱, 展开剂为石油醚(60 $80{ }^{\circ} \mathrm{C}$ )/乙酸乙酯或乙腈/水; ${ }^{1} \mathrm{H}$ NMR 和 ${ }^{13} \mathrm{C}$ NMR 在 BRUKER 公司的 ULTRASHIELD 400 PLUS 或 600 PLUS 核磁共振仪上测定; 质谱在 Waters 公司的 LC/Synapt G2 Q-TOF High Definition Mass Spectrometry 上测定; 所有实验在葛兰素史克(上海)医药研发中心的 工艺开发实验室完成.

\section{2 实验方法}

炔基格氏试剂的制备 ${ }^{[13]}$ : 在氮气流的保护下，用注 射器取一定量的已基溴化镁的四氢呋喃溶液加入到 50 $\mathrm{mL}$ 的两颈瓶中, 再取 1 equiv. 的 3-异丙基硅基乙炔边摚 
拌边逐滴滴加到反应瓶中, 滴加完毕后, 油浴加热到 $50{ }^{\circ} \mathrm{C}$ 反应 $3 \mathrm{~h}$. 反应结束后, 直接投入下一步反应.

偶联产物的合成: 在 $100 \mathrm{~mL}$ 的三颈瓶中加入 0.1 equiv. $\mathrm{FeCl}_{3} 、 0.2$ equiv. Xantphos、10 mL Me-THF 以及 1 equiv. 原料, 在氮气流的保护下用注射器取 2 equiv. 炔 基格氏试剂在 $20 \mathrm{~min}$ 内逐滴滴加入到三颈瓶中, 油浴加 热至 $80{ }^{\circ} \mathrm{C}$ 并回流, 用气相色谱检测仪跟踪检测反应, 3 $\mathrm{h}$ 后反应结束, 用饱和氯化铵溶液淬灭, 乙醚萃取 $(10$ $\mathrm{mL} \times 5)$, 旋蒸后得粗品, 用 $\mathrm{C} 18$ 反相色谱柱 $($ 乙腈/水: 0 $95 \%$ )分离纯化, 得目标产物.

1-环庚基-2-(1',1',1'-三异丙基硅基)-乙炔(1c): 无色 油状液体, 产率 $80 \%$. ${ }^{1} \mathrm{H}$ NMR $\left(400 \mathrm{MHz}^{\circ} \mathrm{CDCl}_{3}\right) \delta$ : $1.01 \sim 1.08\left(\mathrm{~m}, 21 \mathrm{H}, \mathrm{Si}\left(\mathrm{CH}\left(\mathrm{CH}_{3}\right)_{2}\right)_{3}\right), \quad 1.51 \sim 1.55$ and $1.70 \sim 1.78\left(\mathrm{~m}, 6 \mathrm{H}\right.$ and $\left.6 \mathrm{H},\left(\mathrm{CH}_{2}\right)_{6}\right), 2.64 \sim 2.68(\mathrm{~m}, 1 \mathrm{H}$, $\mathrm{CCH}) ;{ }^{13} \mathrm{C} \mathrm{NMR}\left(400 \mathrm{MHz}, \mathrm{CDCl}_{3}\right) \delta: 11.33,18.66$, $25.43,27.81,34.75,32.00,79.79,114.17$. HRMS: 在 ESI 源的高分辨质谱仪上不能检测到该化合物.

1-环己基-2-(1',1',1'-三异丙基硅基)-乙炔(2c): 无色 油状液体, 产率 79\%. ${ }^{1} \mathrm{H}$ NMR (600 MHz, Methanol- $\left.d_{4}\right)$ $\delta: 1.07 \sim 1.09\left(\mathrm{~m}, 21 \mathrm{H}, \mathrm{Si}\left(\mathrm{CH}\left(\mathrm{CH}_{3}\right)_{2}\right)_{3}\right), 1.38 \sim 1.40$ and $1.40 \sim 1.51\left(\mathrm{~m}, 3 \mathrm{H}\right.$ and $\left.3 \mathrm{H},\left(\mathrm{CH}_{2}\right)_{3}\right), 1.73 \sim 1.78(\mathrm{~m}, 4 \mathrm{H}$, $\left.\left(\mathrm{CH}_{2}\right)_{2}\right), 2.48 \sim 2.50(\mathrm{~m}, 1 \mathrm{H}, \mathrm{CCH}) ;{ }^{13} \mathrm{C} \mathrm{NMR}(600 \mathrm{MHz}$, Methanol- $\left.d_{4}\right) \delta: 11.08,17.68,23.99,25.68,29.55,32.43$, 79.06, 113.45. HRMS: 在 ESI 源的高分辨质谱仪上不能 检测到该化合物.

1-环戊基-2-(1',1',1'-三异丙基硅基)-乙炔(3c): 无色 油状液体, 产率 77\%. ${ }^{1} \mathrm{H}$ NMR (400 MHz, Methanol- $\left.d_{4}\right)$ $\delta: 1.02 \sim 1.13\left(\mathrm{~m}, 21 \mathrm{H}, \mathrm{Si}\left(\mathrm{CH}\left(\mathrm{CH}_{3}\right)_{2}\right)_{3}\right), 1.61 \sim 1.67(\mathrm{~m}$, $\left.4 \mathrm{H},\left(\mathrm{CH}_{2}\right)_{2}\right), 1.76 \sim 1.78$ and $1.92 \sim 1.95(\mathrm{~m}, 2 \mathrm{H}$ and $2 \mathrm{H}$, $\left.\left(\mathrm{CH}_{2}\right)_{2}\right), 2.69 \sim 2.78(\mathrm{~m}, 1 \mathrm{H}, \mathrm{CCH}) ;{ }^{13} \mathrm{C} \mathrm{NMR}(600 \mathrm{MHz}$, DMSO- $\left.d_{6}\right) \delta$ : 11.08, 17.65, 24.38, 30.94, 33.80, 78.40, 113.98. HRMS: 在 ESI 源的高分辨质谱仪上不能检测到 该化合物.

1-(4'-氧杂环己环)-2-(1',1',1'-三异丙基硅基)-乙炔 (4c): 无色油状液体, 产率 61\%. ${ }^{1} \mathrm{H}$ NMR $(400 \mathrm{MHz}$, Methanol- $\left.d_{4}\right) \quad \delta: 1.08 \sim 1.13\left(\mathrm{~m}, 21 \mathrm{H}, \mathrm{Si}\left(\mathrm{CH}\left(\mathrm{CH}_{3}\right)_{2}\right)_{3}\right)$, $1.63 \sim 1.67$ and $1.86 \sim 1.91\left(\mathrm{~m}, 2 \mathrm{H}\right.$ and $\left.2 \mathrm{H},\left(\mathrm{CH}_{2}\right)_{2}\right)$, $2.77 \sim 2.81(\mathrm{~m}, 1 \mathrm{H}, \mathrm{CCH}), 3.56 \sim 3.61$ and $3.89 \sim 3.94(\mathrm{~m}$, $2 \mathrm{H}$ and $\left.2 \mathrm{H},\left(\mathrm{CH}_{2}\right)_{2}\right) ;{ }^{13} \mathrm{C} \mathrm{NMR}\left(400 \mathrm{MHz}\right.$, Methanol- $\left.d_{4}\right) \delta$ : $11.03,17.65,32.11,65.56,26.56,80.53,111.41$. HRMS: 在 ESI 源的高分辨质谱仪上不能检测到该化合物.

1-(4'-氮杂环己烷)-2-(1',1',1'-三异丙基硅基)-乙炔 (5c): 无色油状液体, 产率 78\%. ${ }^{1} \mathrm{H}$ NMR $(400 \mathrm{MHz}$, Methanol- $\left.d_{4}\right) \quad \delta: 1.04 \sim 1.16\left(\mathrm{~m}, 21 \mathrm{H}, \mathrm{Si}\left(\mathrm{CH}\left(\mathrm{CH}_{3}\right)_{2}\right)_{3}\right)$, $1.69 \sim 1.74$ and $1.90 \sim 1.94\left(\mathrm{~m}, 2 \mathrm{H}\right.$ and $\left.2 \mathrm{H},\left(\mathrm{CH}_{2}\right)_{2}\right)$, $2.30\left(\mathrm{~m}, 3 \mathrm{H}, \mathrm{CH}_{3}\right), 2.61$ and $2.72(\mathrm{~m}, 1 \mathrm{H}$ and $2 \mathrm{H}, \mathrm{CCH}$ and ), 3.56 3.61 and 3.89 3.94 (m, $2 \mathrm{H}$ and $\left.2 \mathrm{H},\left(\mathrm{CH}_{2}\right)_{2}\right)$; ${ }^{13} \mathrm{C}$ NMR (400 MHz, Methanol- $d_{4}$ ) $\delta: 11.03,17.65,32.11$, 65.56, 26.56, 80.53, 111.41. HRMS (ESI) calcd for $\mathrm{C}_{17} \mathrm{H}_{34}$ $\mathrm{NSi}[\mathrm{M}+\mathrm{H}]^{+}$280.2461, found 280.2462.

1-(4'-对甲苯磺酸基-4'-氮杂环已烷)-2-(1',1',1'-三异 丙基硅基)-乙炔(6c)：黄色固体粉末，产率 85\%. m.p. $60.5{ }^{\circ} \mathrm{C} ;{ }^{1} \mathrm{H}$ NMR (400 MHz, DMSO-d $\left.d_{6}\right) \delta: 0.89 \sim 0.98$ $\left(\mathrm{m}, 21 \mathrm{H},\left(\mathrm{CH}_{3}\right)_{6}\right.$ and $\left.(\mathrm{CH})_{3}\right), 1.73 \sim 1.75\left(\mathrm{~m}, 2 \mathrm{H},\left(\mathrm{CH}_{2}\right)\right)$, $1.88 \sim 1.92\left(\mathrm{~m}, 2 \mathrm{H},\left(\mathrm{CH}_{2}\right)\right), 2.47\left(\mathrm{~m}, 3 \mathrm{H}, \mathrm{CH}_{3}\right), 2.75 \sim 2.77$ $(\mathrm{m}, 1 \mathrm{H}, \mathrm{CH}), 2.90 \sim 2.93\left(\mathrm{~m}, 2 \mathrm{H},\left(\mathrm{CH}_{2}\right)\right), 3.29 \sim 3.31(\mathrm{~m}$, $\left.2 \mathrm{H}, \mathrm{CH}_{2}\right), 7.43 \sim 7.44$ and $7.66 \sim 7.67\left(\mathrm{~m}, 4 \mathrm{H}, \mathrm{C}_{6} \mathrm{H}_{4}\right) ;{ }^{13} \mathrm{C}$ NMR (400 MHz, DMSO- $d_{6}$ ) $\delta: 143.79,132.46,129.48$, 127.54, 109.65, 82.44, 43.18, 30.21, 25.83, 20.14, 17.59, 10.89. HRMS (ESI) calcd for $\mathrm{C}_{23} \mathrm{H}_{38} \mathrm{NO}_{2} \mathrm{SSi}[\mathrm{M}+\mathrm{H}]^{+}$ 420.2393, found 420.2394 .

1-(3'-苯基-4'-氧杂环已环)-2-( $1^{\prime}, 1^{\prime}, 1^{\prime}$-三异丙基硅 基)-乙炔 (7c): 黄色油状液体，产率 71\%. ${ }^{1} \mathrm{H}$ NMR (400 $\left.\mathrm{MHz}, \mathrm{DMSO}-d_{6}\right) \delta: 1.03 \sim 1.04\left(\mathrm{~m}, 21 \mathrm{H}, \mathrm{Si}\left(\mathrm{CH}\left(\mathrm{CH}_{3}\right)_{2}\right)_{3}\right)$, $1.41 \sim 1.45$ and $1.59 \sim 1.62\left(\mathrm{~m}, 2 \mathrm{H}, \mathrm{CH}_{2}\right), 1.86 \sim 1.89$ and $2.06 \sim 2.09\left(\mathrm{~m}, 2 \mathrm{H}, \mathrm{CH}_{2}\right), 2.81 \sim 2.85(\mathrm{~m}, 1 \mathrm{H}, \mathrm{CH}), 3.52 \sim$ $3.56(\mathrm{~m}, 1 \mathrm{H}, \mathrm{CH}), 4.02 \sim 4.04$ and $4.33 \sim 4.35(\mathrm{~m}, 2 \mathrm{H}$, $\left.\mathrm{CH}_{2}\right), 7.26 \sim 7.29$ and $7.34\left(\mathrm{~m}, 5 \mathrm{H}, \mathrm{C}_{5} \mathrm{H}_{5}\right) ;{ }^{13} \mathrm{C} \mathrm{NMR}(400$ $\left.\mathrm{MHz}, \mathrm{DMSO}-d_{6}\right) \delta: 10.0,17.85 \sim 17.95,27.12,31.63$, $66.09,77.09,78.71,111.72,124.95 \sim 125.00,126.59$, 127.53, 141.97. HRMS (ESI) calcd for $\mathrm{C}_{22} \mathrm{H}_{35} \mathrm{OSi}[\mathrm{M}+$ $\mathrm{H}]^{+}$343.2457, found 343.2459.

1-[3'-(3"- 甲基 -4"- 氟苯基)-4'- 氧杂环已环 ]-2$\left(1^{\prime}, 1^{\prime}, 1^{\prime}\right.$-三异丙基硅基)-乙炔 $(\mathbf{8 c})$ : 黄色油状液体，产率 66\%. ${ }^{1} \mathrm{H}$ NMR (400 MHz, DMSO- $\left.d_{6}\right) \delta: 0.99 \sim 1.05(\mathrm{~m}$, $21 \mathrm{H},\left(\mathrm{CH}_{3}\right)_{6}$ and $\left.(\mathrm{CH})_{3}\right), 1.39$ and $1.57 \sim 1.58(\mathrm{~m}, 2 \mathrm{H}$, $\left.\left(\mathrm{CH}_{2}\right)\right), 1.83 \sim 1.86$ and $2.02 \sim 2.05\left(\mathrm{~m}, 2 \mathrm{H},\left(\mathrm{CH}_{2}\right)\right), 2.22$ $\left(\mathrm{m}, 3 \mathrm{H},\left(\mathrm{CH}_{3}\right)\right), 2.79 \sim 2.81(\mathrm{~m}, 1 \mathrm{H},(\mathrm{CH})), 2.47 \sim 3.53(\mathrm{~m}$, $1 \mathrm{H},(\mathrm{CH})), 3.98 \sim 4.02$ and $4.27 \sim 4.29\left(\mathrm{~m}, 2 \mathrm{H},\left(\mathrm{CH}_{2}\right)\right)$, $7.03 \sim 7.08,7.14 \sim 7.17$ and $7.23 \sim 7.25\left(\mathrm{~m}, 3 \mathrm{H},\left(\mathrm{C}_{6} \mathrm{H}_{3}\right)\right)$; ${ }^{13} \mathrm{C}$ NMR (400 MHz, DMSO- $\left.d_{6}\right) \delta: 161.45,159.05(\mathrm{~d}, J=$ $240 \mathrm{~Hz}), 138.88,138.91$ (d, $J=3 \mathrm{~Hz}), 129.25,129.31$ (d, $J=6 \mathrm{~Hz}), 125.24,125.32$ (d, $J=8 \mathrm{~Hz}), 124.17,124.34$ (d, $J=17 \mathrm{~Hz}), 114.81,115.03$ (d, $J=22 \mathrm{~Hz}), 112.72,79.77$, 77.54, 67.16, 40.91, 32.64, 28.13, 18.91, 14.64, 11.06 . HRMS (ESI) calcd for $\mathrm{C}_{23} \mathrm{H}_{36} \mathrm{FOSi}[\mathrm{M}+\mathrm{H}]^{+}$375.2519; found 375.2521 .

1-[3'-(4"-氰基苯基)-4'-氧杂环己环]-2-(1',1',1'-三异 丙基硅基)-乙炔 $(9 \mathrm{c})$ : 半固体糖浆状物质，产率 73\%. ${ }^{1} \mathrm{H}$ NMR (400 MHz, DMSO- $\left.d_{6}\right) \delta: 0.95 \sim 1.02(\mathrm{~m}, 21 \mathrm{H}$, 
$\left(\mathrm{CH}_{3}\right)_{6}$ and $\left.(\mathrm{CH})_{3}\right), 1.31 \sim 1.34$ and $1.57 \sim 1.60(\mathrm{~m}, 2 \mathrm{H}$, $\left.\left(\mathrm{CH}_{2}\right)\right), 1.85 \sim 1.86$ and $2.11 \sim 2.15\left(\mathrm{~m}, 2 \mathrm{H},\left(\mathrm{CH}_{2}\right)\right), 2.81 \sim$ $2.85(\mathrm{~m}, 1 \mathrm{H}, \mathrm{CH}), 3.54 \sim 3.57(\mathrm{~m}, 1 \mathrm{H}, \mathrm{CH}), 4.02 \sim 4.06$ and $4.43 \sim 4.46\left(\mathrm{~m}, 2 \mathrm{H},\left(\mathrm{CH}_{2}\right)\right), 7.53 \sim 7.55$ and $7.79 \sim$ $7.81\left(\mathrm{~m}, 4 \mathrm{H},\left(\mathrm{C}_{6} \mathrm{H}_{4}\right)\right) ;{ }^{13} \mathrm{C} \mathrm{NMR}\left(400 \mathrm{MHz}, \mathrm{CDCl}_{3}\right) \delta$ : $147.63,132.25,126.37,118.86,111.25,110.56,80.85$, 78.20, 67.79, 40.31, 32.42, 28.71, 18.59, 11.15. HRMS (ESI) calcd for $\mathrm{C}_{23} \mathrm{H}_{34} \mathrm{NOSi}[\mathrm{M}+\mathrm{H}]^{+}$368.2410; found 368.2404 .

致谢 感谢上海交通大学与葛兰素史克上海医药研发 中心的联合培养教育项目的支持; 特别感谢葛兰素史克 上海医药研发中心的分析组在定量分析上给予的协助 和有益的讨论.

辅助材料 (Supporting Information) 合成产物的 HNMR 及 ${ }^{13} \mathrm{C}$ NMR 谱图. 这些材料可以免费从本刊网 站(http://sioc-journal.cn/)上下载.

\section{References}

[1] (a) Zhou, J.; Fu, G. C. J. Am. Chem. Soc. 2003, 125, 14726. (b) Fischer, C.; Fu, G. C. J. Am. Chem. Soc. 2005, 127, 4594. (c) Arp, F. O.; Fu, G. C. J. Am. Chem. Soc. 2005, 127, 10482.

[2] (a) Saito, B.; Fu, G. C. J. Am. Chem. Soc. 2007, 129, 9602. (b) Roderiguez, N.; Ramirez de Arellano, C.; Asensio, G.; Medio-Simon, M. Chem. Eur. J. 2007, 13, 4223.

[3] (a) Altenhoff, G.; Wurtz, S.; Glorius, F. Tetrahedron Lett. 2006, 47, 2925.

(b) Caeiro, J.; Perez Sestelo, J.; Sarandeses, L. A. Chem. Eur. J. 2008, 14, 741 .

[4] (a) Ikeda, Y.; Nakamura, T.; Yorimitsu, H.; Oshima, K. J. Am. Chem. Soc. 2002, 124, 6514.

(b) Fujioka, T.; Nakamura, T.; Yorimitsu, H.; Oshima, K. Org. Lett. 2002, 4, 2257

(c) Mizutani, K.; Shinokubo, H.; Oshima, K. Org. Lett. 2003, 5, 3959 .

[5] Wang, H.; Li, L.; Xie, X.; Zhang, R.; Liao, B. CN 103498051, 2014 [Chem. Abstr. 2014, 160, 194031].

[6] Sabir, A.; Debasish, K.; Brindaban, C. R. J. Org. Chem. 2014, 79, 7391.

[7] Deepa, K. D.; Selvakannan, P. R.; Patil, S. K.; Choudhary, V. R.; Bhargava, S. K. Appl. Catal. A: Gen. 2014, 476, 54.

[8] (a) Tamura, M.; Kochi, J. K. Synthesis 1971, 303.

(b) Maillard, B.; Forrest, D.; Ingold, K. U. J. Am. Chem. Soc. 1976,
$98,7024$.

(c) Thoennes, D.; Wesis, E. Chem. Ber. 1978, 111, 3381

(d) Zhang, Y.; Feng, B. N. Chin. J. Org. Chem. 2014, 34, 2406 (in Chinese).

(张艳，冯柏年，有机化学, 2014, 34, 2406.)

(e) Guo, N.; Zhu, S. F. Chin. J. Org. Chem. 2015, 35, 1383 (in Chinese)

(郭娜, 朱守非，有机化学, 2015, 35, 1383.)

[9] (a) Liu, W.; Lei, A. W. Tetrahedron Lett. 2008, 49, 610.

(b) Liu, W.; Cao, H.; Lei, A. W. Angew. Chem., Int. Ed. 2010, 49, 2004.

(c) Wang, H. B.; Wang, L.; Shang, J. S.; Li, X.; Wang, H. Y.; Gui, J.; Lei, A. W. Chem. Commun. 2012, 48, 76.

[10] (a) Rudolph, A.; Lautens, M. Angew. Chem., Int. Ed. 2009, 48, 2656.

(b) Wenkert, E; Han, A. L.; Jonny, C. J. J. Chem. Soc., Chem. Commun. 1988, 975.

(c) Simon, B. B.; David, W. C. M. J. Am. Chem. Soc. 2003, 125, 6046.

[11] (a) Furstner, A.; Leitner, A.; Mendez, M.; Krause, H. J. Am. Chem. Soc. 2002, 124, 13856

(b) Kuzmina, M. O.; Steib, K. A.; Flubacher, D.; Knochel, P. Org. Lett. 2012, 14(18).

(c) Kuzmina, M. O.; Steib, K. A.; Markiewicz, T. J.; Flubacher, D. Angew. Chem., Int. Ed. 2013, 52, 4945.

(d) Nakamura, E.; Yoshikai, N. J. Org. Chem. 2010, 75, 6061.

[12] Hatakeyama, T.; Okada, Y.; Yoshimoto, Y.; Nakamura, M. Angew. Chem., Int. Ed. 2011, 50, 10973.

[13] Cheung, C. W.; Ren, P.; Hu, X. Org. Lett. 2014, 16, 2566.

[14] Zhang, C. X.; Li, N. N.; Li, X.; Chang, H. H.; Liu, Q.; Wei, W. L. Chin. J. Org. Chem. 2014, 34, 81 (in Chinese).

(张聪霞, 李娜娜, 李兴, 常宏宏, 刘强, 魏文珑, 有机化学, 2014, 34, 81.)

[15] (a) Tan, M. X.; Gu, Y. Q.; Luo, X. J.; Zhang, P. Chin. J. Org. Chem. 2015, 35, 781 (in Chinese).

(谭明雄, 顾运琼, 罗旭建, 张培, 有机化学, 2015, 35, 781.) (b) Zhang, B.; Guan, H. X.; Liu, B.; Shi, B. F. Chin. J. Org. Chem. 2014, 34, 1487 (in Chinese).

(张博, 管晗曦, 刘斌, 史炳锋, 有机化学, 2014, 34, 1487.)

(c) Zhang, W. M.; Dai, J. J.; Xu, H. J. Chin. J. Org. Chem. 2015, 35, 1820 (in Chinese).

(张文曼, 戴建军, 许建华, 有机化学, 2015, 35, 1820.)

[16] Eiichi, N.; Takuji, H.; Shingo, I.; Kentaro, I.; Laurean, L.; Nakamura, M. Organic Reactions, Vol. 83, John Wiley \& Sons, Inc., Japan, 2014.

[17] (a) Nakamura, M.; Hirai, A.; Nakamura, E. J. Am. Chem. Soc. 2000. 122, 978.

(b) Yamagami, T.; Shintani, R. T. Org. Lett. 2007, 9, 1045

[18] (a) Nakamura, M.; Matsuo, K.; Inoue, T. Org. Lett. 2003, 5, 1373.

(b) Lautens, M.; Fagnous, K.; Hiebert, S. Acc. Chem. Res. 2003, 36, 48. 